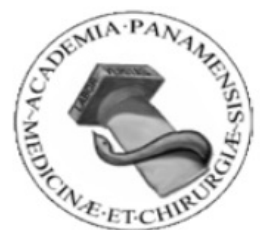

Caso de Interés Radiológico

\title{
Patrón De Empedrado en Hemorragia Alveolar Difusa por Intoxicación con Warfarina
} Paving Pattern in Diffuse Alveolar Hemorrhage Due to Warfarin Intoxication

\section{Paolo Karolyn*, Reyna Rolando**.}

*Médico residente de radiología. **Médico radiólogo, Departamento de radiología,Hospital Santo Tomás, Panamá.

\section{Palabras claves: warfarina, hemoptisis, infiltrados alveolares.}

\section{Key words:}

warfarin, hemoptysis, alveolar infiltrates.

Correspondencia a: Dra Karolyn Paolo

Coreo electrónico: kpaolo30 @hotmail.com

Los autores han declarado que no existen conflictos de intereses con la publicación del presente trabajo.

Se obtuvo el consentimiento informado de todo paciente participante. Los autores declaran autogestion como fuente de financiamiento.

\section{Resumen}

Se presenta un caso de un paciente masculino con antecedente de hipertensión arterial controlada, cardiomiopatía dilatada, fibrilación auricular tratada con warfarina y coartación aórtica en seguimiento, con cuadro de dos días de evolución de hemoptisis y disnea de mínimos esfuerzos, que progresa a vómitos en borlas de café, distrés respiratorio severo y deterioro del estado neurológico, por lo que es intubado de manera inmediata y trasladado a nuestra institución. La radiografía de tórax mostró infiltrados alveolares. En la tomografía de tórax se observó un patrón de empedrado y consolidaciones sugestivos de hemorragia pulmonar por su antecedente clínico.

\section{Abstract}

We present a case of a male patient with a history of controlled arterial hypertension, dilated cardiomyopathy, atrial fibrillation treated with warfarin and aortic coarctation in follow-up, with a two-day history of hemoptysis and minimal effort dyspnea, which progresses to vomiting in tassels of coffee, severe respiratory distress and deterioration of neurological status, so it is intubated immediately and transferred to our institution. Chest x-ray with alveolar infiltrates. In the chest tomography with a crazy paving pattern and consolidations suggestive of pulmonary hemorrhage due to his clinical history.

\section{INTRODUCCIÓN}

Masculino de 49 años con antecedente de hipertensión arterial controlada desde los 35 años, cardiomiopatía dilatada, fibrilación auricular tratada con Warfarina y coartación aórtica en seguimiento, el cual ingresa trasladado del Hospital Regional de Chepo, Panamá, con cuadro de dos días de evolución de hemoptisis y disnea de mínimos esfuerzos, que progresa a vómitos en borlas de café, distrés respiratorio severo y deterioro del estado neurológico, por lo que es intubado de manera inmediata y trasladado a nuestra institución. Al examen físico, el paciente permanece acoplado a ventilación mecánica invasiva, bajo sedación. Lo relevante del laboratorio es: hemoglobina: $5.3 \mathrm{~g} / \mathrm{dl}$, WBC: 20.1 103/ $\mu \mathrm{L}$, tiempo de protrombina $>495$ segundos para un control de 30.7 segundos, INR: 14.6 , fibrinógeno $493 \mathrm{mg} / \mathrm{dl}$.

Estudios de gabinete: Se reporta tomografía cerebral con hematoma subdural derecho y desviación de la línea media. Se le realizan otros estudios de imagen que incluyen radiografía de tórax y angiotomografía de tórax de ingreso.

En la radiografía de tórax de ingreso se visualizan infiltrados pulmonares alveolares bilaterales y marcada cardiomegalia (figura 1). En la Angiotomografía de tórax de ingreso se visualizan zonas de consolidación en ambos campos pulmonares asociadas a patrón en vidrio despulido con engrosamiento de los septos interlobulillares, "patrón de empedrado" (figuras 2, 3, 4).

Estos hallazgos lo asociamos a una etiología vascular vs infecciosa, cardiomegalia y dilatación del tronco de la arteria pulmonar sugestivo de hipertensión pulmonar, secundario a su cardiopatía de base (figura 5). 
Figura 1. Radiografía de tórax de ingreso se visualizan infiltrados pulmonares alveolares bilaterales y marcada cardiomegalia

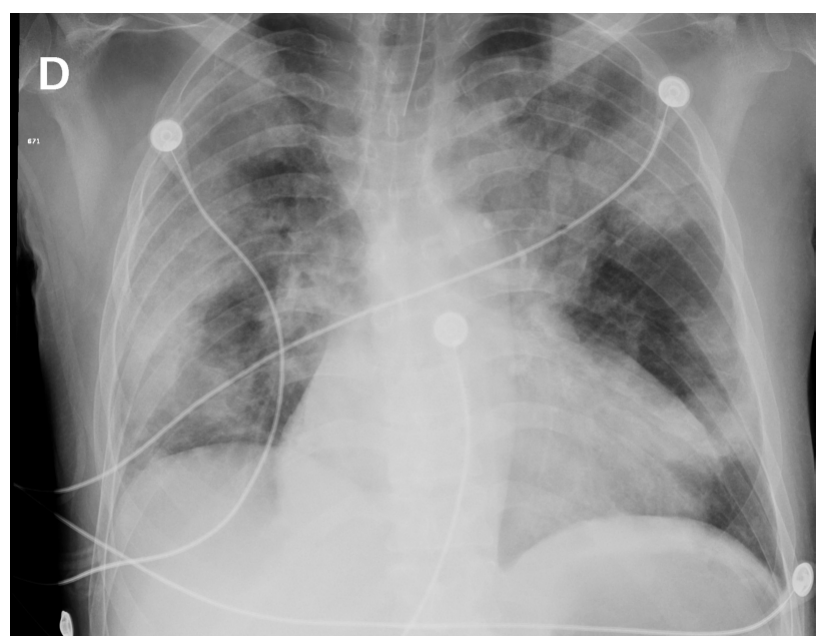

Figura 3. Se visualizan zonas de consolidación en ambos campos pulmonares asociadas a patrón en vidrio despulido con engrosamiento de los septos interlobulillares, "patrón de empedrado"

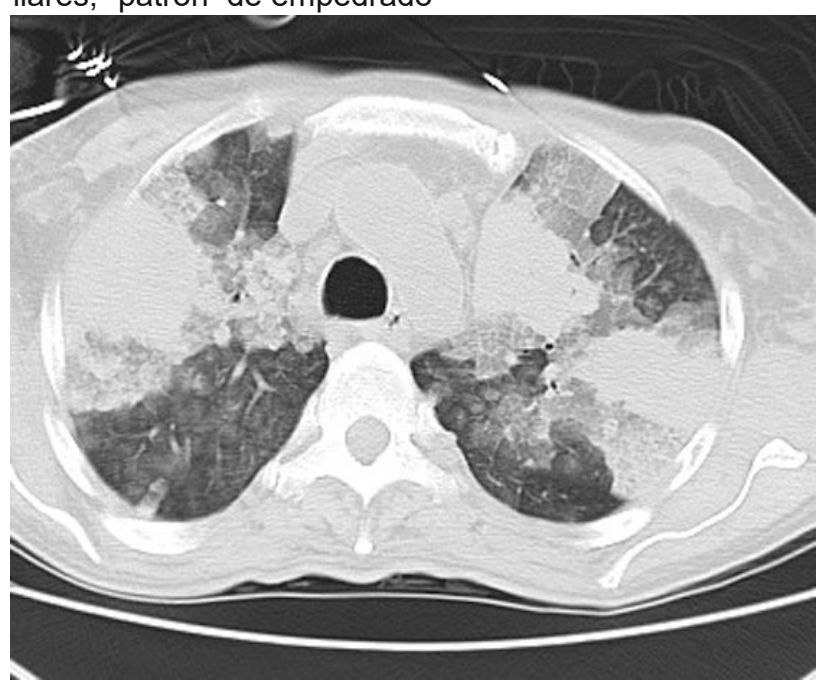

El paciente fallece en unidad de cuidados intensivos dos días después de su ingreso con diagnóstico de hemorragia alveolar difusa secundaria a intoxicación por Warfarina.

\section{DISCUSIÓN}

La hemorragia pulmonar difusa es un síndrome clínico-patológico potencialmente mortal que puede surgir como manifestación de una variedad de enfermedades y causas.

La hemorragia pulmonar presenta dos tipos: hemorragia pulmonar localizada y la hemorragia pulmonar difusa (HPD), que a su vez se subdivide en hemorragia alveolar difusa, cuando el sangrado es difuso y va directo a los espacios alveolares. Se encuentra principalmente asocia-
Figura 2. Zonas de consolidación bilateral, asociadas a patrón en vidrio despulido con engrosamiento de los septos interlobulillares, "patrón de empedrado"

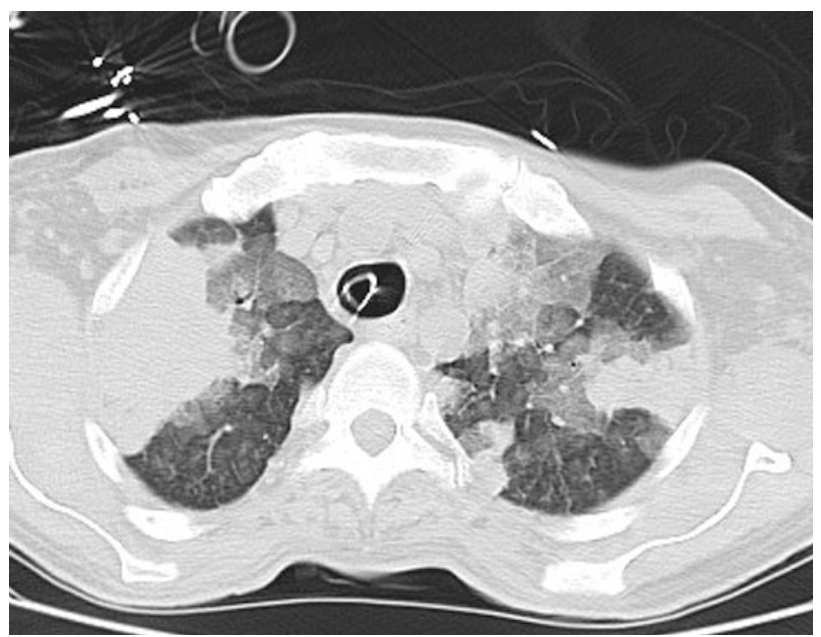

Figura 4. Se visualizan zonas de consolidación en ambos campos pulmonares asociadas a patrón en vidrio despulido con engrosamiento de los septos interlobulillares, "patrón de empedrado"

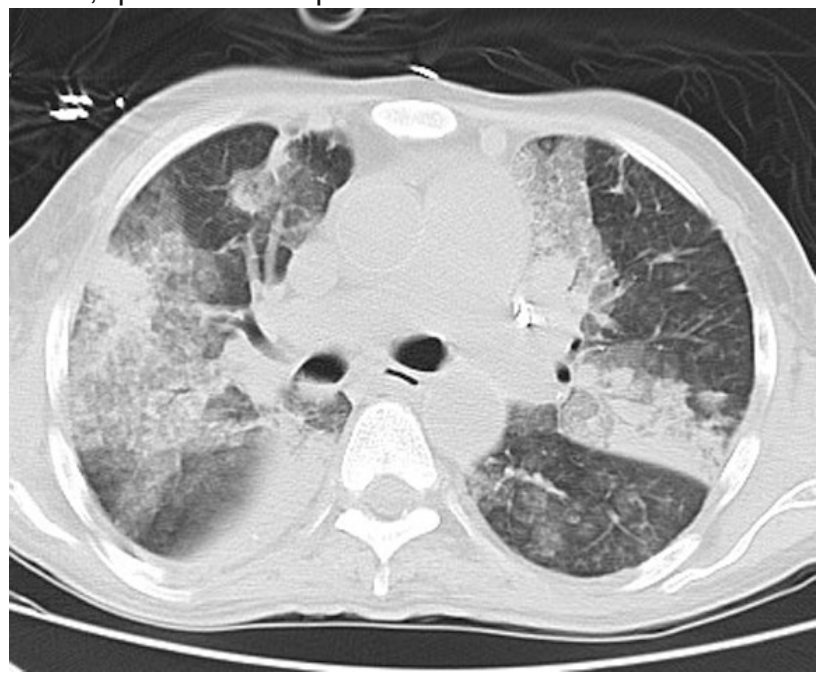

da a vasculitis, pues la capilaritis pulmonar es la lesión más frecuentemente asociada a hemorragia alveolar difusa. Las vasculitis se dividen en: asociadas a ANCA como la granulomatosis de Wegener, la poliangitis microscópica y la vasculitis de Shurg - Strauss y las no asociadas a ANCA como el síndrome de Goodpasture $[1,2,3]$.

También se encuentra relacionada con enfermedades del tejido conectivo como lupus eritematoso sistémico (más comúnmente asociado), enfermedad del tejido conectivo mixto y artritis reumatoide. La hemorragia alveolar difusa se presenta como una complicación rara $(2 \%)$ del lupus, con una tasa reportada de mortalidad de $60 \%$. Típicamente se presenta con los síntomas generales de una vasculitis por LES como falla renal, artritis y rash.

Puede verse como manifestación de procesos infecciosos, desordenes de coagulación, toxicidad a drogas (amiodaro- 
Figura 5. Cardiomegalia y dilatación del tronco de la arteria pulmonar sugestivo de hipertensión pulmonar, secundario a su cardiopatía de base.

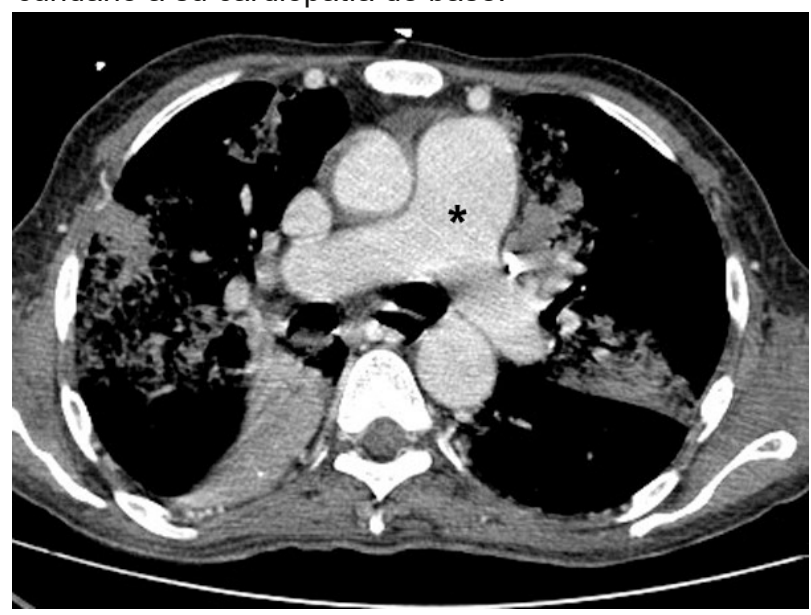

na, nitrofurantoína, entre otros), hemosiderosis idiopática, status post transplante de médula ósea y rara vez como complicación del tratamiento con anticoagulantes, como fue el caso de nuestro paciente. El signo clínico cardinal del HPD es la hemoptisis, sin embargo, esta puede estar ausente en un $33 \%$ de los casos. Otros síntomas frecuentes son la disnea, tos, fiebre y anemia $[4,5,6]$.

El diagnóstico histopatológico diferencial de la hemorragia alveolar difusa incluye enfermedades asociadas con capilaritis pulmonar (infiltración celular de neutrófilos en los capilares y vénulas) y aquellos asociados con vasos normales o hemorragia blanda. El trastorno se debe a un daño en la microcirculación, debido a una lesión en la membrana basal alveolo-capilar con consecuente sangrado en el espacio alveolar, lo que lleva a la acumulación de glóbulos rojos dentro del espacio alveolar y aglomeramiento de macrófagos cargados de hemosiderina en las porciones afectadas del pulmón, luego de 48 a 72 horas.

Los hallazgos imagenológicos de la hemorragia pulmonar difusa dependen de su tiempo de evolución y el contexto clínico del paciente es clave para reconocerla.

En la fase aguda, durante las primeras 24 horas, los estudios radiológicos pueden ser normales en un $20 \%$ de los casos. Cuando están presentes, los hallazgos por radiografía convencional incluyen la presencia de opacidades del espacio aéreo con predominio en porciones centrales y basales, con relativa exclusión de los ápices pulmonares.

Por tomografía computarizada de alta resolución, se pueden observar zonas de atenuación en vidrio despulido, mezcladas con áreas de consolidación, en donde se evidencia la aparente prominencia de los bronquios segmentarios y subsegmentarios, el llamado "Signo del bronquio obscuro". En la fase subaguda, luego de 48 horas, se desarrolla un engrosamiento intra e interlobulillar, el cual superpuesto sobre las zonas de vidrio despulido da un patrón de empedrado ("crazy paving") [2], como pudimos observar en el caso presentado. Dicho patrón inicialmente descrito como signo patognomónico de la proteinosis alveolar, es hoy en día un hallazgo inespecífico, que puede presentarse en un número importante de enfermedades agudas y crónicas, de etiología infecciosa, neoplásica, desordenes sanguíneos y por inhalación, así como enfermedades idiopáticas, representando un reto diagnóstico para el radiólogo. La resolución de estos hallazgos, si no ocurre un nuevo episodio de sangrado, ocurre usualmente luego de 10 días a 2 semanas de un episodio agudo. Este tiempo de resolución es considerablemente más lento que el del edema pulmonar, patrón que tiende a causar confusión con el de la hemorragia alveolar y que debemos considerar dentro del diagnóstico diferencial al encontrar estos hallazgos en estudios de imagen.

En cuanto a los hallazgos entre episodios crónicos recurrentes de sangrado, podemos encontrar opacidades centrilobulillares poco definidas, de tamaño entre 1-3mm, de distribución difusa y sin predominio zonal. También, se visualiza patrón reticular persistente, con panalización y bronquiectasias de tracción, lo que señala depósito intersticial de hemosiderina y fibrosis pulmonar leve. Este patrón suele verse en cualquier tipo de causa de hemorragia alveolar, sin embargo, se ha visto que es particularmente común en pacientes que sufren de hemosiderosis pulmonar idiopática.

Existen otras entidades como el síndrome pulmonar-renal, el cual se manifiesta como una combinación entre la hemorragia alveolar difusa y glomerulonefritis. Siempre debemos incluir dentro de nuestro diagnóstico diferencial las vasculitis asociadas con ANCA como el síndrome de Goodpasture y lupus eritematoso sistémico al encontrarnos con un paciente que debute con este síndrome.

Como complicaciones a largo plazo por episodios repetidos encontramos neumonía organizada y depósito de colágeno en la vía aérea pequeña, que conllevan a fibrosis pulmonar. En el caso del paciente mencionado, debido a las múltiples comorbilidades que presentaba y al estado avanzado en que llegó a la institución, terminó falleciendo a los pocos días de haberse hecho el diagnóstico por métodos de imagen, una de las complicaciones más graves.

\section{CONCLUSIÓN}

La hemorragia alveolar difusa debe ser considerada en pacientes con infiltrados alveolares sin causa aparente particularmente cuando tienen antecedente de alguna enfermedad del tejido conectivo, como el lupus eritematoso sistémico, o que debutan con insuficiencia renal y el uso de anticoagulantes. Si la causa aparente permanece indeterminada luego de una exhaustiva evaluación clínica, que incluye estudios de imagen, serología (para ANCA, anticuerpos anti membrana glomerular, anticuerpos antinucleares e imnunocomplejos circulantes, entre otros) y broncoscopia, se debe considerar biopsia por cirugía. 


\section{REFERENCIAS}

[1] Lichtenberger J, Digurmathy S, Abbott G, Shepard JA, Sharma A. Diffuse Pulmonary Hemorrhage: Clues to the Diagnosis. Curr Probl Diagn Radiol. 2014; 43: 128 - 39.

[2] De Wever W, Meersschaert J, Coolen J, Verbeken E, Verschakelen J. The crazy-paving pattern: a radiological-pathological correlation. Insights Imaging. 2011; 2: 117-32.

[3] Suk Park M. Diffuse Alveolar Hemorrhage. Tuberc Respir Dis. 2013; 74: 151-62.
[4] Cortese G, Nicali R, Placido R, Gariazzo G. Radiological aspects of diffuse alveolar haemorrhage. Radiol Med. 2008; 113: 16-28.

[5] Lara S, Schwarz M. Diffuse Alveolar Hemorrhage. Chest. 2010; 137(5): 1164-71.

[6] Castañer E, Alguersuari A, Gallardo X, Andreu M, Pallardó Y, Mata J, Ramirez J. When to Suspect Pulmonary Vasculitis: Radiologic and Clinical Clues. Radiographics. 2010 